\title{
INNER AMENABILITY AND CONJUGATION OPERATORS
}

\author{
WILLIAM L. PASCHKE ${ }^{1}$
}

\begin{abstract}
It is shown that an infinite discrete group $G$ is inner amenable if and only if the $C^{*}$-algebra generated by the unitaries on $l^{2}(G)$ corresponding to conjugation by $s(s \in G)$ does not contain the projection on the point-mass at the identity.
\end{abstract}

Let $G$ be an infinite discrete group with identity $e$, and let $s \rightarrow L_{s}$ (resp. $R_{s}$ ) denote the left (resp. right) regular representation of $G$ on $H=l^{2}(G)$. For $s \in G$, let $U_{s}=L_{s} R_{s}$, so $U_{s} \xi(t)=\xi\left(s^{-1} t s\right)$ for $\xi \in H$. Write $C^{*}\left(L_{G}, R_{G}\right)$ for the $C^{*}$-algebra generated by the unitaries $L_{s}, R_{s}(s \in G)$, and $C^{*}\left(U_{G}\right)$ for the $C^{*}$-subalgebra of $C^{*}\left(L_{G}, R_{G}\right)$ generated by the unitaries $U_{s}$. Let $\delta$ denote the characteristic function of $\{e\}$, and $P_{\delta}$ the projection on the one-dimensional subspace of $H$ spanned by $\delta$. In [2], using computations from [1], C. A. Akemann and P. A. Ostrand proved that $C^{*}\left(L_{G}, R_{G}\right)$ contains the compact operators when $G$ is the free group on two generators by showing that in this case one has $P_{\delta} \in C^{*}\left(U_{G}\right)$. Our theorem below provides an easier proof (and a generalization) of this result.

Following E. G. Effros [3], we say that $G$ is inner amenable if there is a state $m$ on the $C^{*}$-algebra $l^{\infty}(G)$ such that $m(\delta)=0$ and $m$ is invariant under the automorphisms $T_{s}(s \in G)$ of $l^{\infty}(G)$ defined by $\left(T_{s} f\right)(t)=f\left(s^{-1} t s\right)$. Such an $m$ is called a nontrivial inner mean on $G$. Inner amenability is a considerably weaker condition on $G$ than amenability in the usual sense. The free group on two generators is an easily accessible example of a group which is not inner amenable (see [3]). Inner amenability and the behavior of $C^{*}\left(U_{G}\right)$ are related by the following theorem.

THEOREM. The group $G$ is inner amenable if and only if $P_{\delta} \notin C^{*}\left(U_{G}\right)$.

Proof. First suppose that $P_{\delta} \notin C^{*}\left(U_{G}\right)$. Since $U_{s} \delta=\delta$ for each $s \in G$, it follows that $P_{\delta} X=X P_{\delta}=(X \delta, \delta) P_{\delta}$ for every $X \in C^{*}\left(U_{G}\right)$, so $C^{*}\left(U_{G}\right)+$ $\mathbf{C} P_{\delta}$ is a ${ }^{*}$-algebra. We claim that it is norm-closed, and hence a $C^{*}$-algebra. [Suppose $X_{n}+z_{n} P_{\delta} \rightarrow Y$ in norm, with $X_{n} \in C^{*}\left(U_{G}\right)$ and $z_{n} \in \mathbf{C}$. The sequence $\left\{z_{n}\right\}$ must be bounded (for otherwise we could pass to a subsequence and assume $\left|z_{n}\right| \rightarrow \infty$, forcing $z_{n}^{-1} X_{n}+P_{\delta} \rightarrow 0$ and thereby contradicting $P_{\delta} \notin C^{*}\left(U_{G}\right)$ ), so we may assume that $z_{n} \rightarrow z \in \mathbf{C}$ and hence

Received by the editors September 16, 1977 and, in revised form, November 29, 1977.

AMS (MOS) subject classifications (1970). Primary 46L05.

Key words and phrases. $C^{*}$-algebra, discrete group, inner amenable.

${ }^{1}$ Research partially supported by a grant from the National Science Foundation. 
$X_{n} \rightarrow Y-{ }_{z} P_{\delta}$. We conclude that $Y \in C^{*}\left(U_{G}\right)+\mathbf{C} P_{\delta}$.] Our hypothesis permits us to define a linear functional $\phi$ on $C^{*}\left(U_{G}\right)+C P_{\delta}$ by $\phi\left(X+z P_{\delta}\right)=$ $(X \delta, \delta)$. Notice that

$$
\phi\left(\left(X+z P_{\delta}\right)^{*}\left(X+z P_{\delta}\right)\right)=\left(X^{*} X \delta, \delta\right) \geqslant 0,
$$

so $\phi$ is a state. Extend $\phi$ to a state $\psi$ on the algebra $B(H)$ of bounded operators on $H$. Since $\psi\left(U_{s}\right)=1$, we see that $I-U_{s}$ belongs to the left and right kernels of $\psi$ for every $s \in G$ and hence $\psi\left(U_{s} X U_{s}^{*}\right)=\psi(X)$ for every $X \in B(H)$. For $f \in l^{\infty}(G)$, let $\pi(f)$ be the corresponding multiplication operator on $H$. Since $U_{s} \pi(f) U_{s}^{*}=\pi\left(T_{s} f\right)$ and $\pi(\delta)=P_{\delta}$, the state $\psi \circ \pi$ on $l^{\infty}(G)$ is a nontrivial inner mean for $G$, as required.

For the converse direction, suppose that $G$ is inner amenable. The "convergence to invariance" argument in [3] yields a net $\left\{\xi_{\alpha}\right\}$ of unit vectors in $H$ with $\xi_{\alpha}(e)=0$ for every $\alpha$ and

$$
\lim _{\alpha}\left\|U_{s} \xi_{\alpha}-\xi_{\alpha}\right\|=0
$$

for every $s \in G$. Let $\psi$ be any $w^{*}$-limit state of the net of vector states on $B(H)$ corresponding to the net $\left\{\xi_{\alpha}\right\}$. We have $\psi\left(U_{s}\right)=1(s \in G)$ and $\psi\left(P_{\delta}\right)=0$. By applying $\psi$ and the vector state corresponding to $\delta$, one sees that the norm of the difference of $P_{\delta}$ and any finite linear combination of the $U_{s}^{\prime}$ 's must be at least $\frac{1}{2}$, so $P_{\delta} \notin C^{*}\left(U_{G}\right)$.

\section{REFERENCES}

1. C. A. Akemann and P. A. Ostrand, Computing norms in group $C^{*}$-algebras, Amer. J. Math. 98 (1976), 1015-1047.

2. On a tensor product $C^{*}$-algebra associated with the free group on two generators (preprint).

3. E. G. Effros, Property $\Gamma$ and inner amenability, Proc. Amer. Math. Soc. 47 (1975), 483-486.

Department of Mathematics, University of Kansas, Lawrence, Kansas 66045 